\title{
Semiautomatic quantitation of macrophages in human renal biopsy specimens in proteinuric
} states

\author{
P N Furness, L Rogers-Wheatley, K P G Harris
}

Department of Pathology, Leicester General Hospital, Gwendolen Road, Leicester LE5 4PW P N Furness

Department of Nephrology K P G Harris

Department of Pathology, Leicester Royal Infirmary, PO

Box 65, Leicester

LE2 7LX

L Rogers-Wheatley

Correspondence to: Dr P N Furness.

Accepted for publication 5 November 1996

\begin{abstract}
Aims-To develop and validate a rapid and economical semiautomated approach to the measurement of immunostainable tissue components which is applicable to routine diagnostic practice. To apply this approach to the measurement of macrophages in renal biopsy specimens in nephrotic states, as protein in the renal tubules may induce macrophage infiltration, and the morphology of macrophages in tissue sections does not lend itself to cell counting.

Methods-Macrophages were identified by immunostaining with a panmacrophage marker, followed by digital image capture and analysis using a macro procedure written for the freeware image analysis program NIH-Image.

Results-The method was rapid, robust and accurate to within the limits imposed by sampling error inherent in the use of small needle biopsy specimens. Very few macrophages are found in normal kidney (mean volume fraction $( \pm 95 \%$ confidence limits) $0.04 \%(0.02 \%)$ ) but infiltration of macrophages was detected in minimal change nephropathy $(0.29 \%(0.12 \%))$ and in membranous glomerulonephritis $(0.42 \%(0.11 \%))$. A statistically significant correlation was found between macrophage volume fraction and weight of proteinuria in minimal change nephropathy but not in membranous glomerulonephritis. Correlations were found in both diseases between macrophage volume fraction and serum creatinine at time of biopsy.

Conclusions-The equipment is inexpensive and measurement takes less than one minute per biopsy specimen. The results indicate that macrophage infiltration is part of the pathological process in minimal change nephropathy and membranous glomerulonephritis. The correlation with creatinine at time of biopsy suggests that renal impairment in minimal change nephropathy may result from infiltration by immunologically active cells and not merely from haemodynamic changes in
\end{abstract}

nephrons. However, the correlation is not close, indicating that the relation between macrophage infiltration and disease severity is not a simple one. ( Clin Pathol 1997;50:118-122)

Keywords: macrophage; minimal change; membranous; glomerulonephritis; nephrotic morphometry.

The role of the macrophage in the induction of fibrosis is becoming clearer in a variety of disease processes. In the kidney, most emphasis has been placed on macrophage infiltration in the glomerulus, with consequent production of cytokines and induction of excess deposition of extracellular matrix. The renal interstitium has been studied comparatively rarely, despite the acknowledged importance of interstitial fibrosis in renal impairment, ${ }^{12}$ and despite the observation of foam cells in the interstitium in biopsy specimens from patients with heavy proteinuria from a variety of causes.

Recently, there have been several attempts to predict the course of chronic human renal disease from the renal biopsy specimen by measuring the numbers of myofibroblasts in the interstitium. This has been quite successful in IgA nephropathy ${ }^{3}$ and in membranous glomerulonephritis, ${ }^{4}$ both of which are normally rather unpredictable in their course. However, the method used, manual counting of cells after immunocytochemical detection, is laborious and for this reason alone is unlikely to gain wide acceptance in routine diagnosis.

In general terms, one may postulate the following as a typical sequence of events in a fibrotic reaction: an insult leads to macrophage infiltration, which results in cytokine production, which stimulates proliferation and activity of matrix producing cells (such as myofibroblasts). ${ }^{56}$ If this is accepted, it seems possible that quantitation of interstitial macrophages rather than myofibroblasts might produce an earlier and potentially more sensitive marker of the severity of the insult to the kidney, although resolution may occur if the infiltration is short-lived. Therefore, we sought to measure the level of macrophage infiltration in renal biopsy specimens from adult patients with idiopathic membranous nephropathy, with minimal change nephropathy and, as a control group, 


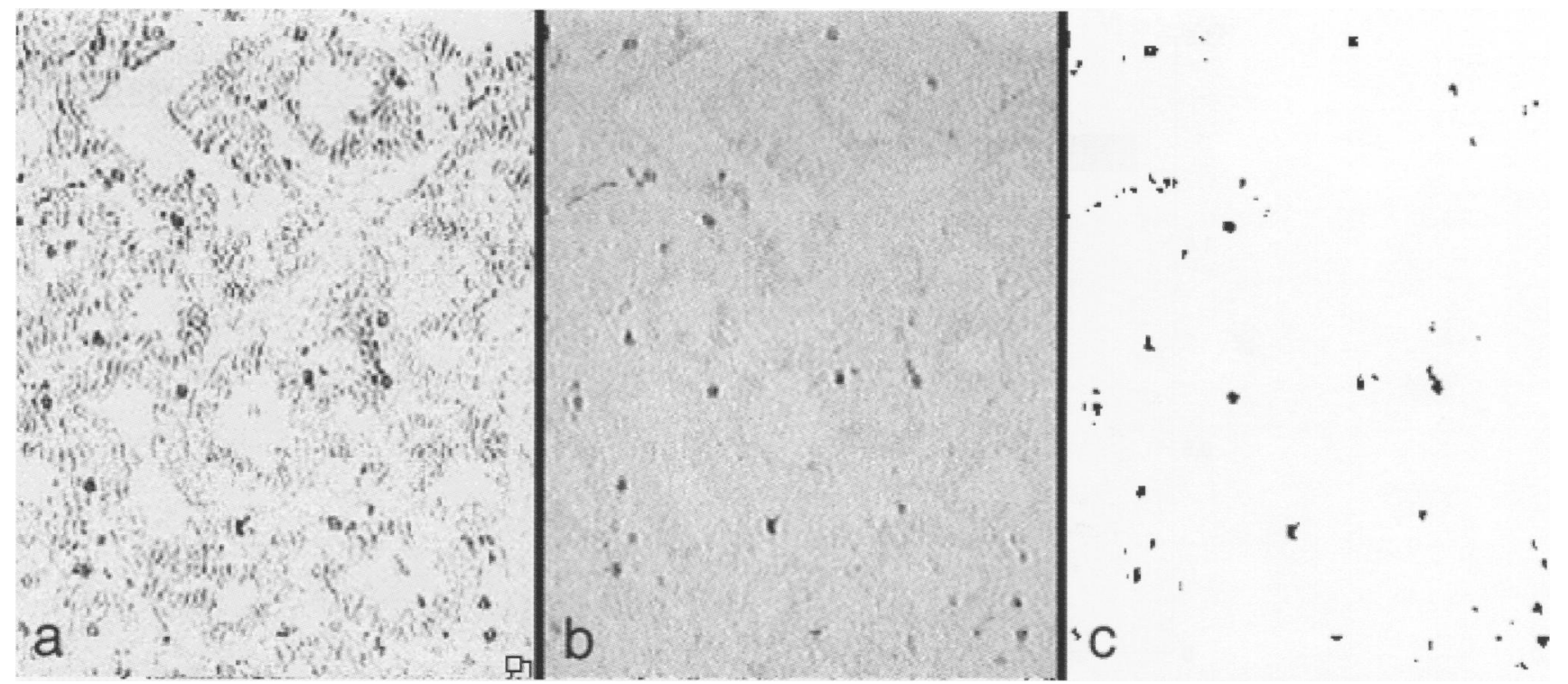

Figure 1 A digital greyscale image of an area of relatively heavy macrophage infiltration, immunostained for macrophages using PGM1, but without counterstain. (a) An image captured with the condenser diaphragm closed, to indicate the location of the (unstained) tubules. Such an image is not part of the normal analysis process. (b) The same field captured with normal illumination, before thresholding. (c) Image (b) after thresholding. When thresholding has been completed, the computer can calculate the percentage of pixels which are black almost instantaneously; this is taken as representative of the volume fraction of macrophages in this area of the biopsy specimen. The software maintains a 'running mean' as multiple fields from a single specimen are examined.

biopsy specimens from patients with microscopic haematuria and no detectable renal abnormality. These diseases were chosen as they are conventionally described as not having an inflammatory component. Furthermore, it has been suggested that heavy proteinuria might lead to interstitial fibrosis by directly inducing the release of macrophage chemoattractants by 'damaged' tubular epithelial cells.

Published studies in this field have almost invariably used cell counting to measure the density of cellular infiltrates. This is laborious, and not applicable to routine practice. Furthermore, immunostaining of macrophages in renal biopsy specimens shows cells with complex cytoplasmic extensions ramifying through the interstitium, making definition of what constitutes a single cell very difficult and somewhat subjective. To facilitate this study, and to provide a tool for further work, we sought to develop and validate a rapid computer-assisted method for measurement of components of renal biopsy specimens which would eliminate the need for laborious cell counting, and which could be applied in routine practice.

\section{Methods}

Sequential renal biopsy specimens were identified from the files of the Histopathology Department at Leicester General Hospital representing each of the following three categories: normal (34 cases); minimal change nephropathy ( 31 cases); and membranous nephropathy (43 cases). Cases were only accepted if the diagnoses were supported by light microscopy, immunocytochemistry for immunoglobulins and complement, and electron microscopy. Cases in the 'normal' category were all biopsies performed for the investigation of microscopic haematuria without proteinuria in which al investigations of the biopsy specimen had shown no abnormality. The cases were not specifically matched for age, but no paediatric cases were included.
Clinical data collected included serum creatinine concentrations and the 24 hour urine protein output at the time of biopsy.

\section{IMMUNOSTAINING}

Paraffin wax sections $(3 \mu \mathrm{m})$ were dewaxed, and treated with $6 \% \mathrm{H}_{2} \mathrm{O}_{2}$ to block endogenous peroxidase. Sections were exposed to $0.1 \%$

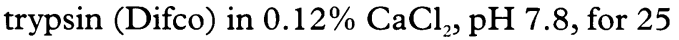
minutes at $37^{\circ} \mathrm{C}$. A standard indirect immunoperoxidase method was used with PGM1 (Dako), a pan-macrophage marker, as the primary antibody applied overnight at $4^{\circ} \mathrm{C}$ at a dilution of 1 in 50 in TRIS-buffered saline. A counterstain was omitted in order to facilitate subsequent image analysis.

\section{IMAGE ANALYSIS}

Sections were viewed on a Zeiss photomicroscope III with a JVC TK $120 \mathrm{E}$ video camera attached. The camera was linked with a Y/C cable to the in-built framegrabber board of an Apple Macintosh 7100/80 AV microcomputer, and images were imported directly to the freeware image analysis program NIH-Image*, using the 'Plug-in digitizer' software Photoshop-compatible plug-in.

Sequential images were grabbed using the $\times 10$ objective, moving along the central line of each biopsy specimen from one end of the available cortex to the other, without overlapping. The presence of glomeruli in the fields was ignored. Renal medulla was not measured.

To calculate the volume fraction of the macrophages, each image was converted to greyscale, and then a threshold was applied at a level which distinguished between the stained macrophages and the unstained background.

*The image analysis program NIH-Image was written by Wayne Rasband (e-mail: wayne@helix.nih.gov.) and is distributed as freeware (at: http://rsb.info.nih.gov/nihimage/ or by anonymous ftp from zippy.nimh.nih.gov.). 


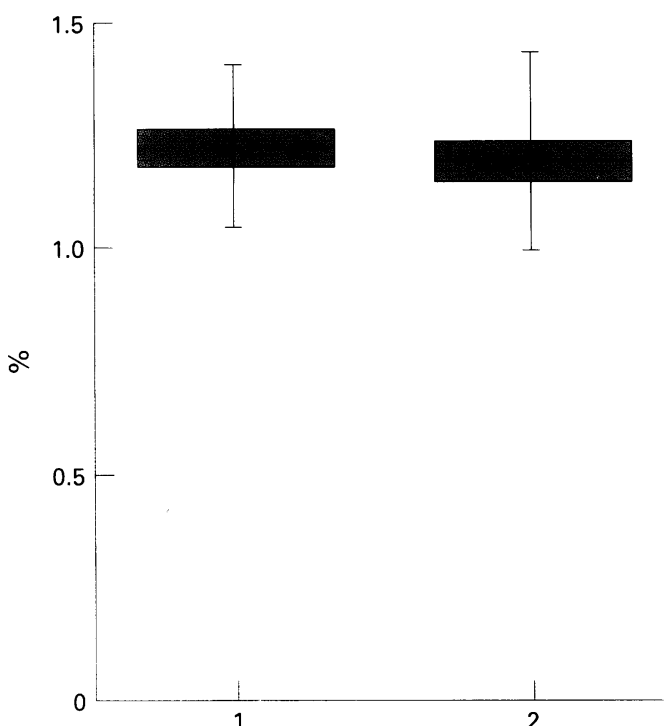

Figure 2 Results of measuring macrophage volume fraction using 10 serial sections, all stained on separate occasions. Mean (central line), range (vertical line) and quartiles (shaded bar) are shown. Results are shown for measurements made on two separate occasions.

The proportion of black to white pixels in the image (excluding the edges) was then calculated as a percentage. As sequential fields were assessed, a running mean of the macrophage area fraction was maintained automatically. Processing of a typical image is illustrated in fig 1 .

A set of macros was written in the NIHImage programming language to automate this process, such that sequential fields can be measured with just two keystrokes per field. These macros are available as a simple text file, free by e-mail on request to pnf1@le.ac.uk.

METHOD VALIDATION

To test the reproducibility of the image analysis method, 10 sequential sections were cut from a single renal biopsy specimen. These were immunostained with PGM1 on 10 separate occasions, each occasion separated by at least three days. The volume fraction of macrophages in the resulting set of 10 slides was then measured, and the measurements were repeated the following day.

STATISTICAL ANALYSIS

The regression coefficients were calculated as part of the graphing process by the program CA-Cricket graph III; $p$ values for these linear correlations were calculated using Systat. Comparisons between the mean macrophage volume fractions in normal biopsy specimens and those in the disease groups were made using Student's $t$ test (unpaired), as calculated by Microsoft Excel; 95\% confidence limits were also calculated using Excel.

\section{Results}

METHOD VALIDATION

The results of measurements of 10 replicate sections are shown in fig 2 . The means, quartiles and maximum/minimum figures show little variation between the two occasions on which they were measured. The overall variation is considerably less than one might

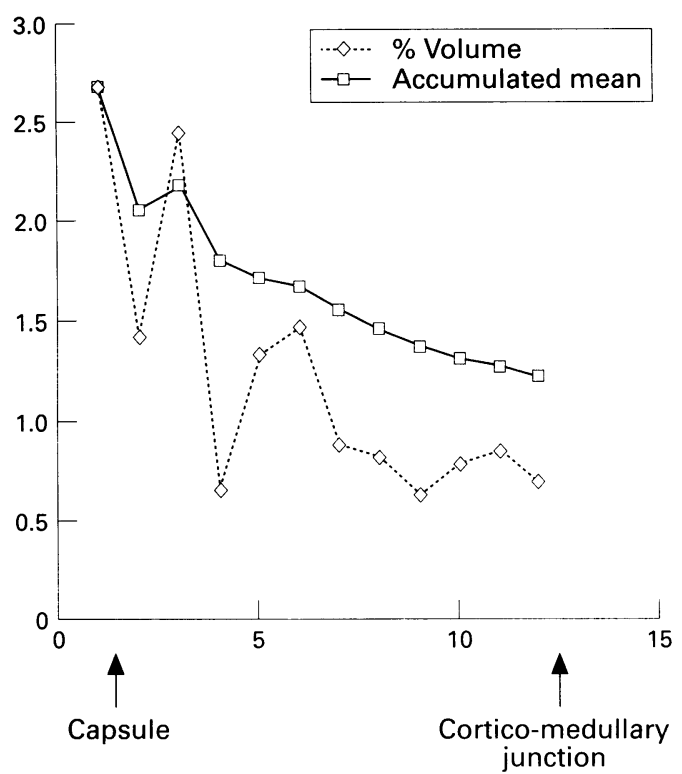

Figure 3 Measurements of volume fraction in adjacent non-overlapping fields of a single biopsy specimen (membranous glomerulonephritis), running from renal capsule (left) to cortico-medullary junction (right). Dotted line, actual measurements; solid line, accumulated mean. It is evident that in this case there is a gradient of macrophage infiltration across the cortex, which is of considerably greater magnitude than the measurement error as shown in fig 2.

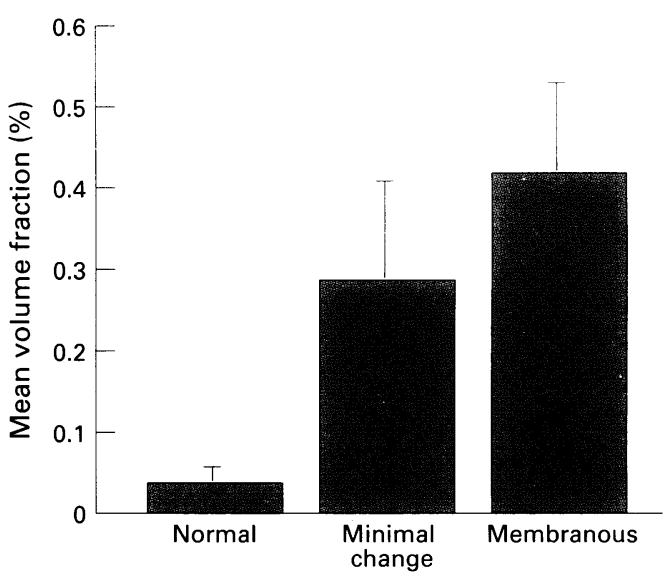

Figure 4 Macrophage volume fractions in normal biopsy specimens, in minimal change nephropathy and in membranous glomerulonephritis. Error bars represent 95\% confidence limits.

expect with a quantitative immunocytochemical method, and we felt use of the method in the main part of the study was justified.

In most biopsy specimens, the exact depth of the cortical tissue could not be judged. In a few specimens, however, the renal capsule and cortico-medullary junction were both visible. Such cases were too few for statistical analysis, but there seemed to be a gradient of macrophage numbers which decreased from outer to inner cortex (fig 3 ). This finding probably justifies further study; if confirmed, it suggests that sampling error, rather than measurement error, is likely to be the biggest source of random variation with this method.

MACROPHAGE VOLUME FRACTIONS

The mean macrophage volume fractions are shown in fig 4. Macrophage infiltration in minimal change nephropathy and in membranous nephropathy was significantly greater 

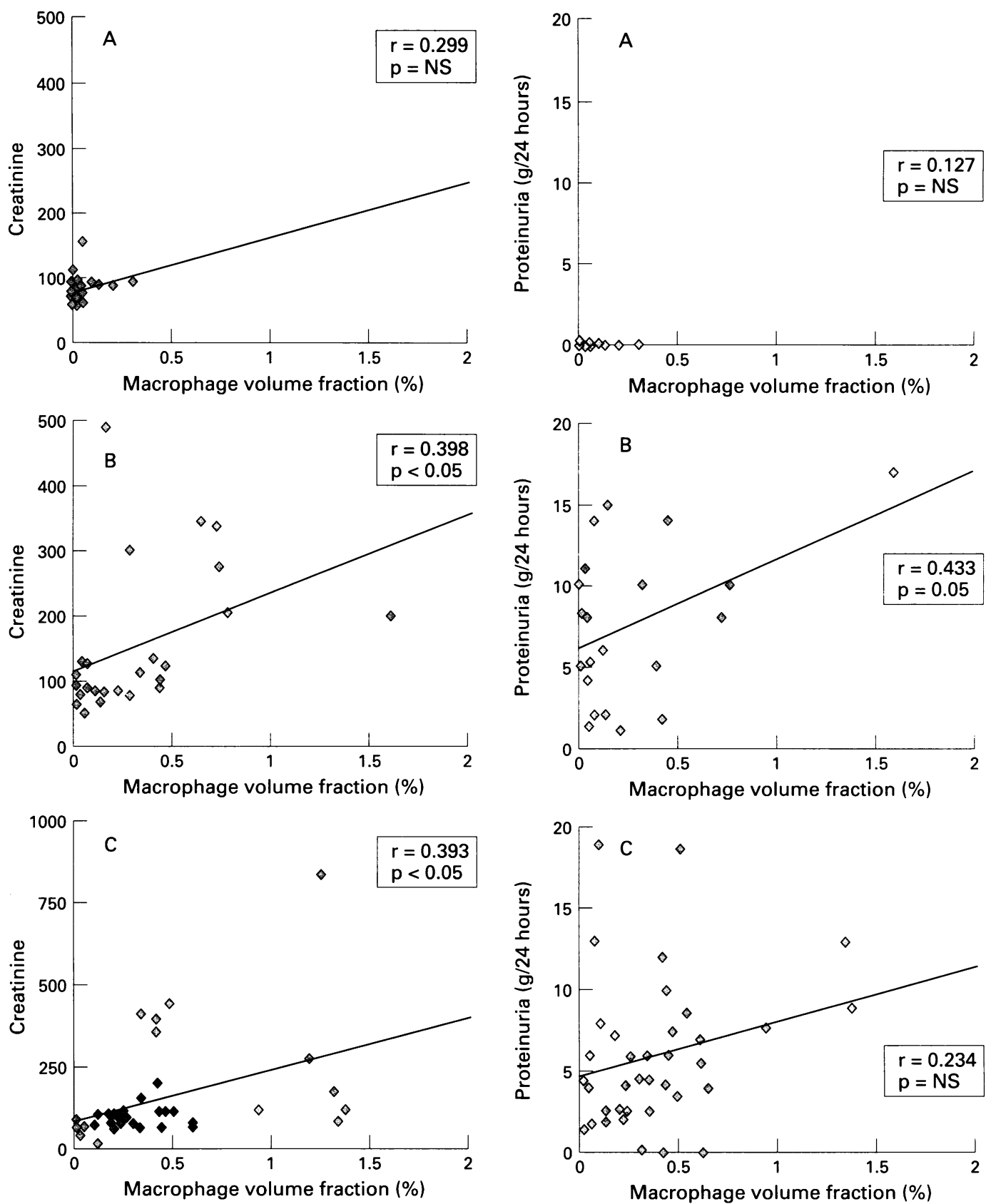

Figure 5 Scatter graphs showing correlations between macrophage volume fraction and creatinine at time of biopsy in (A) normal specimens, in (B) minimal change nephropathy and in (C) membranous glomerulonephritis.

than in normal controls $(p<0.0001)$. Although the mean level of macrophage infiltration in membranous glomerulonephritis was greater than in minimal change disease, the difference was not statistically significant $(p=0.13)$.

There was a significant correlation between macrophage volume fraction and serum creatinine at the time of biopsy in membranous nephropathy and minimal change disease (fig 5). A significant correlation was also found with the 24 hour urine protein output in minimal change disease (fig 6). However, it is obvious from the scatter plots that the regression value is low and the correlation is poor. These correlations have only just reached significance, even though our automated measuring system permitted the analysis of relatively large numbers of specimens.

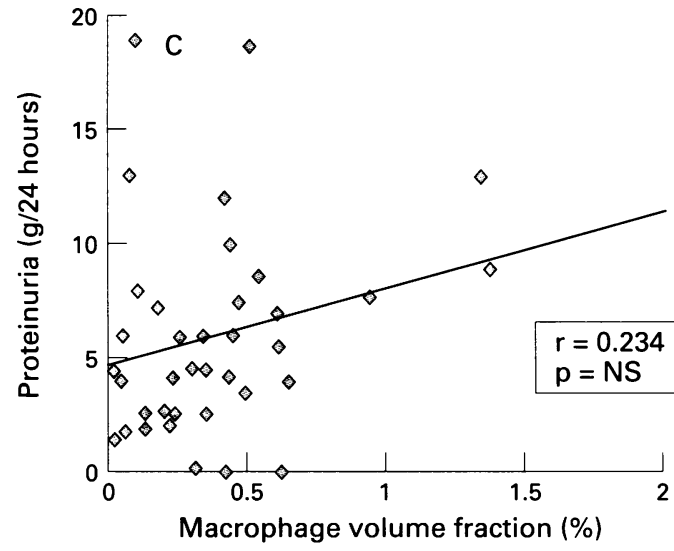

Figure 6 Scatter graphs showing correlations between macrophage volume fraction and 24 hour urine protein output immediately before biopsy in $(A)$ normal specimens, in (B) minimal change nephropathy and in $(C)$ membranous glomerulonephritis.

\section{Discussion}

These results confirm that a significant interstitial infiltrate of macrophages is a part of the pathological process in membranous nephropathy and in minimal change nephropathy. The existence of a correlation between macrophage volume fraction and serum creatinine in both diseases implies that immunological mechanisms may be involved in the reduced glomerular filtration rate which is commonly seen at presentation of both diseases. This is not surprising in the case of membranous glomerulonephritis; but in minimal change nephropathy, the presence of interstitial macrophages is not widely recognised as part of the disease process. Indeed, one argument which has been used against the puromycin aminonucleoside (PAN) model of minimal change nephropathy is that PAN induces an interstitial 
infiltrate of macrophages, on which the renal impairment seen in the model depends. ${ }^{8}$ Our results suggest that this criticism is invalid, and also argue against the widely held view that any renal impairment in minimal change nephropathy has a haemodynamic cause, ${ }^{9}$ reflecting circulation volume changes in nephrosis, rather than an immunological one. Furthermore, it shows that the presence of a moderately heavy interstitial infiltrate of macrophages does not necessarily indicate the presence of an irreversible fibrotic process, as minimal change nephropathy rarely, if ever, progresses to renal fibrosis.

A close correlation has previously been reported between macrophage infiltration and proteinuria in mesangial proliferative glomerulonephritis with mesangial IgM. ${ }^{10}$ Tubular expression of monocyte chemoattractant protein-1 (MCP-1) is known to be increased in membranous glomerulonephritis and to correlate with the interstitial macrophage infiltrate. ${ }^{11}$ It has been suggested that the mere presence of large quantities of filtered protein in the tubules represents an insult to the tubular epithelium which can result in chemokineinduced macrophage infiltration. ${ }^{12}$ This certainly seems to be the case in the protein overload model of proteinuria in the rat. ${ }^{13} \mathrm{We}$ have shown that MCP-1 is released by cultured tubular epithelial cells after the addition of protein to the medium bathing the apical surface of the cells.

However, macrophage infiltration is induced in many animal models of fibrotic disease, in the kidney and other organs. ${ }^{14}$ This includes the fibrosis caused by ureteric obstruction, where proteinuria is not an issue. ${ }^{15}{ }^{16}$ Of course, we do not know for how long our patients had proteinuria before biopsy was performed; variation in this time may account for some of the variation in macrophage numbers, but it seems likely that other aspects of the disease process are involved in the variation we have seen. If assessment of macrophages in renal biopsy specimens is to be relevant to the severity and progression of disease, it is evident that we should be measuring macrophage function rather than simply their presence. Assessment of cytokines such as tumour growth factor $\beta$ may be more promising. ${ }^{17}$

There has been considerable interest recently in quantitative or semiquantitative approaches to predicting the rate of disease progression from renal biopsy specimens, both native ${ }^{34}$ and transplant. ${ }^{18-20}$ Most of these studies have used either semiquantitative scoring on a scale of 0 to 3 by a 'blinded' observer, or laborious manual morphometry, or cell counting. Having previously undertaken a study requiring point-counting, ${ }^{21}$ we have been very favourably impressed with the method de- scribed here, which can be applied to any tissue component that can be localised by immunostaining. The high speed and low expense $\mathrm{e}^{22}$ of the method mean that if the clinical significance of such methods can be demonstrated, it will be easy to convert this information into a routine quantitative assessment-a feature currently lacking in most diagnostic histopathology reports.

Part of this work was supported by a grant from the Medical Research Council to PNF.

1 Risdon RA, Sloper J, DeWardener H. Relationship between renal function and histological changes found in renal biopsy specimens from patients with persistent glomerular nephritis. Lancet 1968;ii:363-6.

2 Schainuck L, Striker G, Luther R, Benditt E. Structuralfunctional correlations in renal disease. II. The correlations. Hum Pathol 1970;1:631-41.

3 Goumenos DS, Brown CB, Shortland J, el Nahas AM. Myofibroblasts, predictors of progression of mesangial IgA nephropathy? Nephrol Dial Transplant 1994;9:1418-25.

4 Roberts IS, Burrows C, Shanks JH, Venning M, McWilliam LJ. Interstitial myofibroblasts; predictors of progression in membranous nephropathy [abstract]. F Pathol 1996;178S: 44A.

5 Eddy AA. Interstitial macrophages as mediators of renal fibrosis. Experimental Nephrology 1995;3:76-9.

6 Furness PN. Extracellular matrix and the kidney. $f$ Clin Pathol 1996;49:355-9.

7 Burton C, Walls J, Harris K. Tubular MCP-1 production; a mechanism for proteinuria-induced interstitial inflammation [abstract]. Proc Eur Dial Trans Assoc 1996;33:71A.

8 Harris K, Lefkowith J, Klahr S, Schreiner G. Essential fatty acid deficiency ameliorates acute renal dysfunction in the rat after the administration of the aminonucleoside of puromycin. $\mathcal{F}$ Clin Invest 1990;86:1115-23.

9 Lieberthal W, Levinsky NG. Acute clinical renal failure. In: Seldin DW, Giebisch G, eds. The kidney: physiology and pathophysiology. New York: Raven Press, 1992:3181-225.

10 Garcia del Moral R, Gomez-Morales M, Cortes V, Aguayo ML, Gigosos RL, Lardelli P, et al. Mononuclear cell subsets in IGM mesangial proliferative glomerulonephritis subsets in IgM mesangial proliferative glomerulonephritis A comparative study with minimal change nephrotic syndrome and immunonegative mesangial proliferative

11 Prodjosudjadi W, Gerritsma JS, van Es LA, Daha MR, BruProdjosudjadi W, Gerritsma JS, van Es LA, Daha MR, Bru-
ijn JA. Monocyte chemoattractant protein-1 in normal and diseased human kidneys: an immunohistochemical analysis. Clin Nephrol 1995;44:148-55.

12 Burton C, Harris K. The role of proteinuria in the progression of chronic renal failure. Am $\mathcal{F}$ Kidney Dis 1996;27:76575.

13 Eddy AA, Giachelli CM. Renal expression of genes that promote interstitial inflammation and fibrosis in rats with protein-overload proteinuria. Kidney Int 1995;47:1546-57.

14 El Nahas AM. Pathways to renal fibrosis. Exp Nephrol 1995; 3:71-5.

15 Schreiner G, Harris K, Purkerson M, Klahr S. The immunological aspects of acute ureteral obstruction: Charimmunological aspects of acute ureteral obstruction: Characterisation, localisation and kinetics of the immune
infiltrate in the kidney. Kidney Int 1988;34:487-93.

16 Diamond JR. Macrophages and progressive renal disease in experimental hydronephrosis. Am $\mathcal{F}$ Kidney Dis 1995;26: 133-40.

17 Yamamoto T, Noble NA, Miller DE, Border WA. Sustained expression of TGF-beta 1 underlies development of progressive kidney fibrosis. Kidney Int 1994;45:916-27.

18 Isoniemi $\mathrm{H}$, Taskinen $\mathrm{E}$, Hayry P. Histological chronic allograft damage index accurately predicts chronic renal graft damage index accurately predicts chronic

19 Dimeny E, Wahlberg J, Larsson E, Fellstrom B. Can histopathological findings in early renal allograft biopsies histopathological findings in early renal allograft biopsies

20 Rush DN, Jeffery JR, Gough J. Sequential protocol biopsies in renal transplant patients: repeated inflammation is associated with impaired graft function at 1 year. Transplan Proc 1995;27:1017-18.

21 McCulloch TA, Harper SJ, Donnelly PK, Moorhouse J, Bel $\mathrm{PR}$, Walls $\mathrm{J}$, et al. Influence of nifedipine on interstitial fibrosis in renal transplant allografts treated with cyclosporin A. F Clin Pathol 1994;47:839-42.

22 Furness PN. Morphometry in pathology [letter]. 7 Pathol $1995 ; 177: 441$. 